\title{
Accelerated tumor growth under intermittent hypoxia is associated with hypoxia-inducible factor-1-dependent adaptive responses to hypoxia
}

\author{
Dae Wui Yoon ${ }^{1, *}$, Daeho So ${ }^{2, *}$, Sra Min ${ }^{1,2}$, Jiyoung Kim ${ }^{1,2}$, Mingyu Lee ${ }^{1,2}$, Roza \\ Khalmuratova ${ }^{1}$, Chung-Hyun Cho ${ }^{1,2,3}$, Jong-Wan Park ${ }^{1,2,3,4}$ and Hyun-Woo Shin ${ }^{1,2,3,4,5}$ \\ ${ }^{1}$ Obstructive Upper Airway Research (OUaR) Laboratory, Department of Pharmacology, Seoul National University College of \\ Medicine, Seoul 03080, Korea \\ ${ }^{2}$ Department of Biomedical Science, Seoul National University Graduate School, Seoul 03080, Korea \\ ${ }^{3}$ Ischemic/Hypoxic Disease Institute, Seoul National University College of Medicine, Seoul 03080, Korea \\ ${ }^{4}$ Cancer Research Institute, Seoul National University College of Medicine, Seoul 03080, Korea \\ ${ }^{5}$ Department of Otorhinolaryngology-Head and Neck Surgery, Seoul National University Hospital, Seoul 03080, Korea \\ *These authors have contributed equally to this work \\ Correspondence to: Hyun-Woo Shin, email: charlie@snu.ac.kr \\ Keywords: obstructive sleep apnea, cancer, hypoxia-inducible factor, intermittent hypoxia, hypoxia adaptation \\ Received: February 28, $2017 \quad$ Accepted: May 29, $2017 \quad$ Published: June 27, 2017 \\ Copyright: Yoon et al. This is an open-access article distributed under the terms of the Creative Commons Attribution License 3.0 \\ (CC BY 3.0), which permits unrestricted use, distribution, and reproduction in any medium, provided the original author and source \\ are credited.
}

\section{ABSTRACT}

Mounting evidence has revealed a causative role of intermittent hypoxia (IH) in cancer progression in mouse models of obstructive sleep apnea (OSA), but most studies have focused on the effects of IH following tumor implantation using an exposure to single IH frequency. Thus, we aimed to investigate 1) the potential effect of IH on the initial tumor growth in patients with OSA without an interaction with other mechanisms induced by IH in mice and 2) the influence of the IH frequency on tumor growth, which were tested using pre-conditioning with IH (Pre-IH) and 2 different IH frequencies, respectively. Pre-IH was achieved by alternatively maintaining melanoma cells between normoxia ( $10 \mathrm{~min}, 21 \% \mathrm{O}_{2}$ ) and hypoxia ( $50 \mathrm{~min}, 1 \% \mathrm{O}_{2}$ ) for 7 days (12 cycles per day) before administering them to mice. The conditions for IH-1 and IH-2 were 90 s of $12 \% \mathrm{FiO}_{2}$ followed by 270 s of $21 \% \mathrm{FiO}_{2}$ (10 cycles/h), and $90 \mathrm{~s}$ of $12 \%$ $\mathrm{FiO}_{2}$ and $90 \mathrm{~s}$ of $21 \% \mathrm{FiO}_{2}(20 \mathrm{cycles} / \mathrm{h}$ ), respectively, for $8 \mathrm{~h}$ per day. Tumor growth was significantly higher in the Pre-IH group than in the normoxia group. In addition, the IH-2 group showed more accelerated tumor growth compared to the normoxia and IH-1 groups. Immunohistochemistry and gene-expression results consistently showed the up-regulation of molecules associated with HIF-1 $\alpha$-dependent hypoxic adaptation in tumors of the Pre-IH and IH-2 groups. Our findings reveal that IH increased tumor progression in a frequency-dependent manner, regardless of whether it was introduced before or after in vivo tumor cell implantation.

\section{INTRODUCTION}

Obstructive sleep apnea (OSA) is a common nocturnal respiratory symptom affecting $2 \%$ to $5 \%$ of adults in the general population $[1,2]$. Patients with OSA undergo repetitive partial or complete obstruction of the upper airway during sleep. OSA results in frequent sleep-fragmentation events, intrathoracic negativepressure swings, and intermittent hypoxia (IH). Those characteristics are regarded as potential pathophysiological factors for the development or worsening of several chronic diseases associated with OSA, including 
cardiovascular diseases, metabolic diseases, and neuropsychiatric problems.

The severity of OSA is typically determined by the number of hypopnea (partial obstruction) and apnea (complete obstruction) events per h of sleep, referred to as the apnea-hypopnea index (AHI) and categorized as mild $(5 \leq \mathrm{AHI}<15)$, moderate $(15 \leq \mathrm{AHI}<30)$, or severe $(30 \geq \mathrm{AHI})$. Numerous previous findings have shown that the risk or severity of chronic diseases and pathological conditions are dose-dependently associated with the OSA severity [3-6].

Several recent epidemiological and experimental findings have shown a significant association between OSA and cancer risk. The Wisconsin Sleep Cohort Study was performed to examine the association between OSA and cancer-specific mortality [7]. The results confirmed that significantly higher hazard ratios (HRs) for cancer mortality occurred in the severe-OSA group (HR, 4.8; $95 \%$ CI, 1.7-13.2) compared to non-OSA subjects, with a trend toward increasing cancer mortality according to OSA severity. In addition, the results of a Spanish multicenter study showed a significant relationship between OSA and cancer incidence [8]. Interestingly, it appears that specific cancer types are particularly associated with OSA. According to a study performed to analyze the health insurance data of 5.6 million individuals, the risks for pancreatic cancer, kidney cancer, and melanoma were significantly higher in OSA patients [9], while the risks for breast, colorectal, and prostate cancer were lower in OSA patients than in a control group. Although these epidemiological studies revealed a positive association between OSA and cancer, several confounding variables (e.g., age, obesity, and lifestyle) make it difficult to clarify the direct causal effect of OSA on cancer risk.

It has been well documented that hypoxia frequently occurs in solid tumors. This is because the density of the blood vessels is not sufficient to support the perfusion of whole tumors, which rapidly proliferate. Thus, some tumor cells receive an insufficient oxygen supply. To overcome or adapt to hypoxic conditions resulting from the lack of oxygen supply, tumors promote formation of their own vascular network via hypoxia-inducible factor-1 (HIF-1), a master regulator of expression of several genes under hypoxia (e.g., angiogenesis, metabolism, metastasis, or glucose uptake) $[10,11]$. Two types of hypoxia occur within tumor tissues, namely chronic hypoxia and $\mathrm{IH}$. Accumulating evidences consistently reported that the tumor interior is close to $\mathrm{IH}$ conditions with spatial and temporal changes in hypoxia, as evidenced by dynamic IH measurements [12] and indirect measurements of fluctuating tumor blood flow [13]. It has been reported that HIF-1 is more accumulated in cells exposed to $\mathrm{IH}$ than those undergoing chronic hypoxia [14, 15]. It is noteworthy that HIF-1 has been regarded as a therapeutic target for cancer progression, and the level of vascular endothelial growth factor (VEGF), which is a downstream of HIF-1 correlated with tumor weights in a murine model of OSA [16].

$\mathrm{IH}$ conditions mimicking $\mathrm{O}_{2}$-saturation profiles with recurrent apneic events seen in patients with OSA, promoted tumor progression and aggressiveness in mouse xenograft models [17-19]. However, these studies mostly used experimental protocols wherein mice were exposed to $\mathrm{IH}$ following tumor cell injection and were then monitored for longitudinal tumor changes; thus, only the effect of IH on tumor progression could be explored. Moreover, despite indications that the severity of OSA is involved in the worsening or development of morbidities associated with OSA, most studies have used only a single IH frequency in the study design. Thus, these studies did not elucidate whether tumor growth or malignancy were influenced by the frequency of IH. With this in mind, we established 2 different experimental protocols. First, tumor cells were preconditioned by $\mathrm{IH}$ prior to injection into mice without additional $\mathrm{IH}$ exposure during in vivo tumor progression (Figure 1A). This protocol may explain the potential effect of IH on the initial tumor growth in patients with OSA, without an interaction with other mechanisms induced by IH in mice. Second, tumor cells maintained under normoxia were injected into mice, and then 2 different protocols involving either $10 / \mathrm{h}$ or $20 / \mathrm{h}$ of continuous IH were used with the mice during tumor progression (Figure 1B). The 2 different IH conditions of $10 / \mathrm{h}$ and $20 / \mathrm{h}$ represent mild and moderate OSA, respectively. Melanoma cells were selected for this study because a higher risk of melanoma has been reported in patients with OSA [9].

\section{RESULTS}

\section{Pre-intermittent hypoxia (Pre-IH) conditioning promoted in vivo tumor growth}

Preconditioning of melanoma cells by $\mathrm{IH}$ was achieved by alternatively maintaining melanoma cells between normoxia (10 $\mathrm{min}, 21 \% \mathrm{O}_{2}$ ) and hypoxia (50 min, $1 \% \mathrm{O}_{2}$ ) for 7 days (12 cycles per day). Non-conditioned cells were continuously maintained in the normoxic chamber. Mice inoculated with Pre-IH-conditioned melanoma cells exhibited more accelerated tumor growth than those of the no-conditioning $(\mathrm{N})$ group did at day 19 post-tumor injection (left panel in Figure 2A and 2B). Tumor weights were also significantly higher in the Pre$\mathrm{IH}$ group than in the $\mathrm{N}$ group (Figure 2C). No significant difference in body weight was observed between the 2 groups throughout the experimental period (right panel in Figure 2A).

We examined whether Pre-IH conditioning would induce alterations in HIF- $1 \alpha$, HIF- $2 \alpha$, or pyruvate dehydrogenase kinase 1 (PDK1; a downstream effector of HIF) expression during the adaptation of cells to hypoxia (Supplementary Figure 1A). Cells exposed to 
7 days of $\mathrm{N}$ or Pre-IH conditioning were lysed and used for western blot analysis. HIF-1 $\alpha$ was rarely expressed in both $\mathrm{N}$ and Pre-IH cells. HIF-2 $\alpha$ expression was slightly higher in the Pre-IH group than in the N group. Pre-IHconditioned cells showed higher PDK1 expression than did the $\mathrm{N}$ cells, indicating an accumulated hypoxic burden of Pre-IH-conditioned cells. Prior to performing cell cycle analysis and proliferation assays, cells grown under $\mathrm{N}$ or Pre-IH conditions for 7 days were further incubated overnight under normoxic conditions. The proportion

A

\section{Pre-IH conditioning to cells}

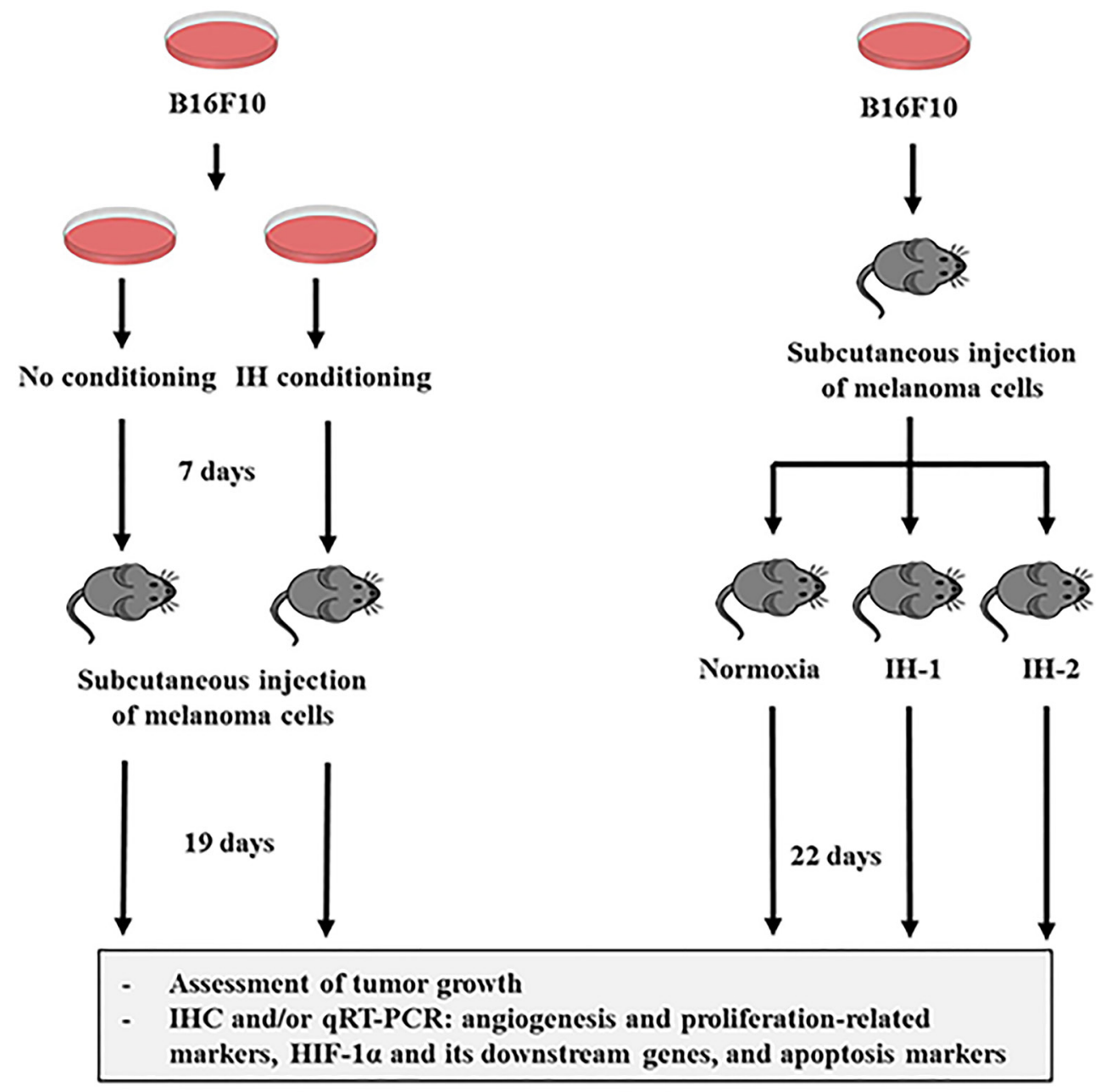

B of DNA at different cell cycle stages (G1/G0, S, and G2/M) was assessed by flow cytometry in N- and Pre-IHconditioned cells. As shown in Supplementary Figure 1D, Pre-IH conditioning did not affect any cell cycle stage in B16F10 cells. In BrdU-based cell-proliferation assays, no difference was observed in proliferation between $\mathrm{N}$ and Pre-IH-conditioned cells (Supplementary Figure 1E). These results suggested that 7 days of Pre-IH conditioning did not alter the proliferative potential of tumor cells in vitro.

\section{IH exposure of tumor-bearing mice}

Figure 1: Schematic representation of the experimental protocols used in this study. (A) Mouse melanoma B16F10 cells were preconditioned by intermittent hypoxia (Pre-IH) with alternating cycles of $1 \% \mathrm{O}_{2}$ for $50 \mathrm{~min}$, or $21 \% \mathrm{O}_{2}$ for $10 \mathrm{~min}(12$ cycles/day for 84 total cycles), or maintained under normoxia (N; no conditioning) for 7 days before subcutaneous injection into C57BL/6 mice. Then, the tumor-bearing mice were maintained in ambient room air for 19 days without additional exposure to intermittent hypoxia (IH). (B) B16F10 cells maintained under normoxia were subcutaneously injected into C57BL/6 mice, and then the mice were randomly divided into the N, $\mathrm{IH}-1$, and $\mathrm{IH}-2$ groups. Mice in these groups were then subjected to normoxia, $\mathrm{IH}-1\left(90 \mathrm{~s}\right.$ of $12 \% \mathrm{FiO}_{2}$ followed by $270 \mathrm{~s}$ of $21 \% \mathrm{FiO} 2[10$ cycles/h]), or IH-2 ( $90 \mathrm{~s}$ of $12 \% \mathrm{FIO}_{2}$ and $90 \mathrm{~s}$ of $\left.21 \% \mathrm{FIO}_{2}[20 \mathrm{cycles} / \mathrm{h}]\right)$ conditions for 22 days. Tumor growth was assessed by measuring estimated tumor volumes during the experimental period. Tumors were excised from the mice and processed for immunohistochemical staining and quantitative real-time polymerase chain reaction analysis. 


\section{Pre-IH conditioning increased HIF-1 $\alpha-$ dependent adaptive responses to hypoxia in tumors of xenografted mice}

We performed immunohistochemical staining (IHC) to examine whether Pre-IH conditioning affects adaptive responses to hypoxia in mouse tumors. Nuclear HIF-1 $\alpha$ expression was significantly higher in tumors from mice inoculated with Pre-IH-conditioned cells than in tumors from mice inoculated with $\mathrm{N}$-conditioned cells (Figure 3A and 3B). Given that HIF-1 $\alpha$ is a well-known transcription factor for VEGF, which stimulates angiogenesis, we examined whether angiogenesis was increased in tumors of the Pre-IH group. Expression of cluster of differentiation 31 (CD31), an endothelial cell and angiogenesis marker, was significantly up-regulated in tumors in the Pre-IH group compared to those in the $\mathrm{N}$ group. Proliferation of tumor cells also increased in tumors of the Pre-IH group, as demonstrated by up-regulation of the proliferating cell nuclear antigen (PCNA). There was no difference in the number of terminal deoxynucleotidyl transferase dUTP nick end labeling (TUNEL)-positive nuclei between the 2 groups, implying that accelerated tumor growth in the Pre-IH group was not due to increased cell survival, but was due to the promotion of cell proliferation. To confirm the up-regulated expression of HIF- $1 \alpha$ in IHC, we performed quantitative real-time polymerase chain reaction (qRT-PCR) and examined the expression of HIF-1 $\alpha$ (Supplementary Figure 1A) and its downstream target genes. As shown in Figure 3C, expression of HIF$1 \alpha$ downstream target genes such as $V E G F-A$, glucose transporter 1 (GLUT1), and PDK1 increased in tumors of the Pre-IH group, compared to those of the $\mathrm{N}$ group. However, no significant difference was observed in the expression level of HIF-1 $\alpha$ itself between the Pre-IH and $\mathrm{N}$ groups (Supplementary Figure 1B).

\section{Post-IH conditioning increased in vivo tumor growth in a frequency-dependent manner}

Next, we examined whether IH exposure after tumor xenografting could affect tumor growth. As depicted in Figure 4A, oxygen-saturation $\left(\mathrm{SaO}_{2}\right)$ profiles in freely moving mice exposed to $\mathrm{N}$, IH-1, or $\mathrm{IH}-2$ conditions were obtained by mouse pulse oximetry. The $\mathrm{SaO}_{2}$ nadir under the IH-1 condition ranged from 67 to $73 \%$.

A

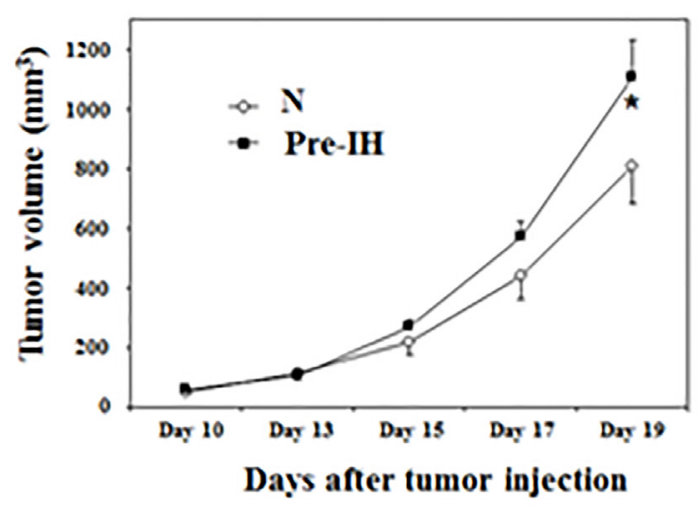

B

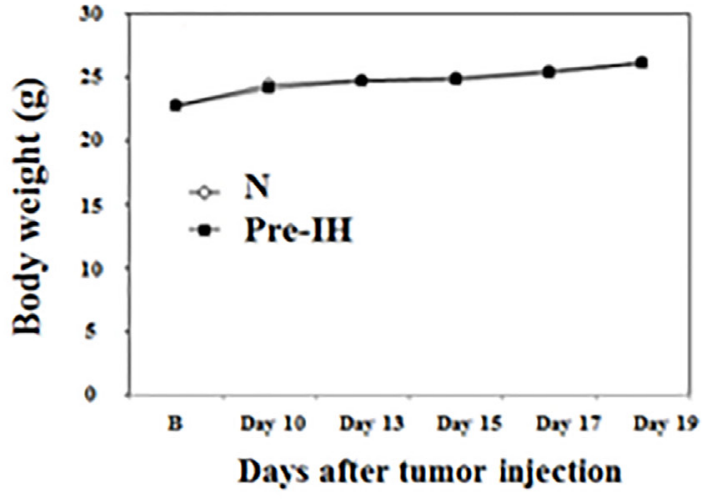

C
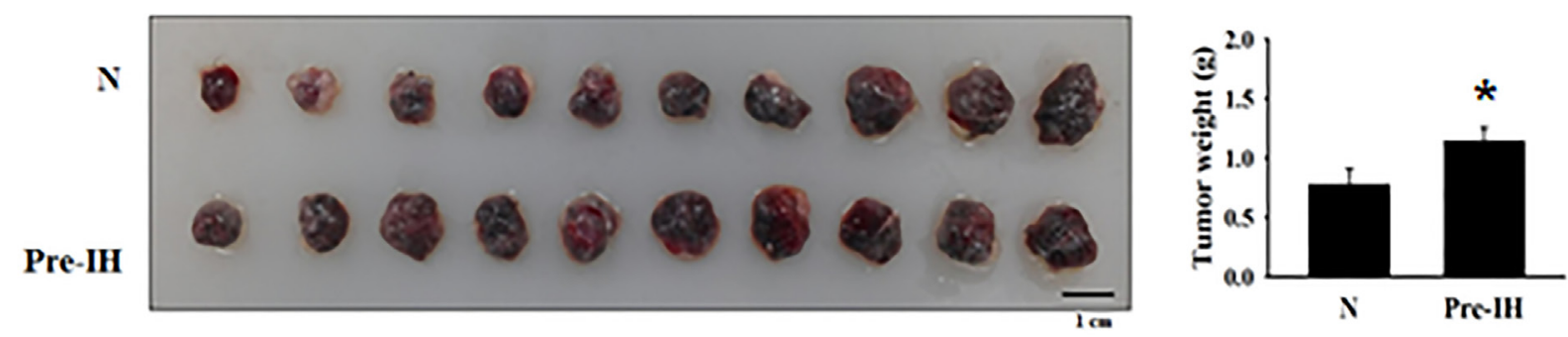

Figure 2: Tumor growth in mice in the no-conditioning $(\mathrm{N})$ and pre-intermittent, hypoxia-conditioning (Pre-IH) groups. (A) Changes in tumor volumes and body weights. Tumor volumes were measured every 2 or 3 days starting from day 10 posttumor injection. At day 19, tumor volumes in mice in the Pre-IH group were significantly higher than those in the $\mathrm{N}$ group ( $\mathrm{n}=10$ /group). (B) Comparison of tumor sizes from mice in the $\mathrm{N}$ and Pre-IH groups. (C) Comparison of tumor weights in the $\mathrm{N}$ and Pre-IH groups. Data are presented as the mean \pm S.E.M. ${ }^{*} P<0.05$ compared to $\mathrm{N}$ group, as determined by the Mann-Whitney U test. 
A slightly lower nadir of $\mathrm{SaO}_{2}$ was observed under the IH-2 condition (from 62 to $68 \%$ ). At day 18 after injecting melanoma cells, the tumor volumes were higher in the $\mathrm{IH}-2$ group than in the $\mathrm{N}$ and $\mathrm{IH}-1$ groups (upper panel in Figure 4B). No differences in body weights were observed among the groups during the entire experimental period (lower panel in Figure 4B). Tumor weights were also significantly higher in the IH-2 group than in N and IH-1 groups (Figure 4C and 4D). Tumor volumes and weights did not differ between the $\mathrm{N}$ and $\mathrm{IH}-1$ groups throughout the experimental period and at the time of sacrifice. One mouse each in the N and IH-2 groups and 3 mice in IH-1 group died during the experimental period, possibly due to tumor-induced death, which occurs frequently in B16F10-bearing mice [20]. We checked whether the loss of mice affected the final results regarding the tumor size and found that the mouse tumor volumes at the time of death were similar with the corresponding group's average tumor volume (data not shown). Thus, our results were

A

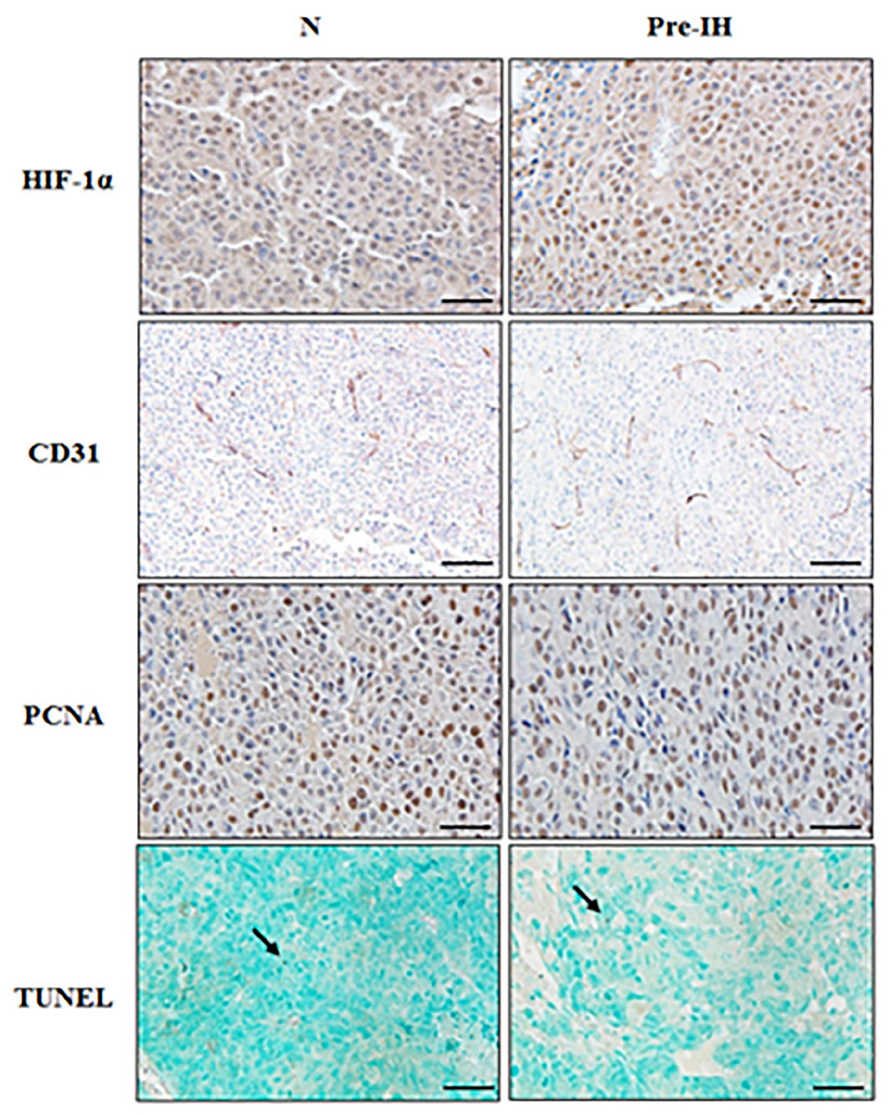

likely not affected by the reduction in data due to the lost mice.

\section{Increases in HIF-1 $\alpha$-dependent adaptive responses to hypoxia were frequency-dependent}

IHC was performed to examine the expression levels of HIF-1 $\alpha$, CD31, and PCNA, as well as the number of TUNEL-positive nuclei in tumors from mice subjected to $\mathrm{N}, \mathrm{IH}-1$, or $\mathrm{IH}-2$ conditions. Tumors in the IH-2 group showed significantly increased expression of those proteins versus the $\mathrm{N}$ and $\mathrm{IH}-1$ groups, although the number of TUNEL-positive cells did not differ (Figure 5A and 5B). qRT-PCR analysis showed more increased expression of HIF- $1 \alpha$ downstream target genes and the endothelial cell marker CD31 in tumors of the IH-2 group than in those of the $\mathrm{N}$ and $\mathrm{IH}-1$ groups (Figure $5 \mathrm{C}$ ), which confirmed the increased transcriptional activity of HIF$1 \alpha$ in the IH-2 group. However, we did not observe a
B
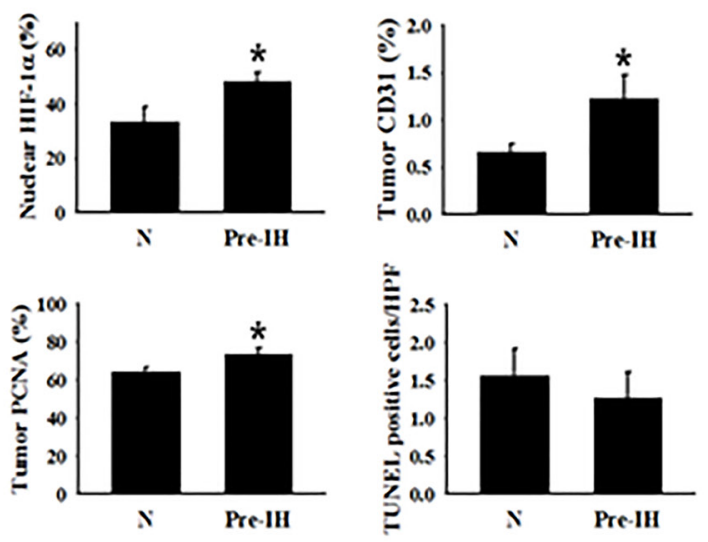

C
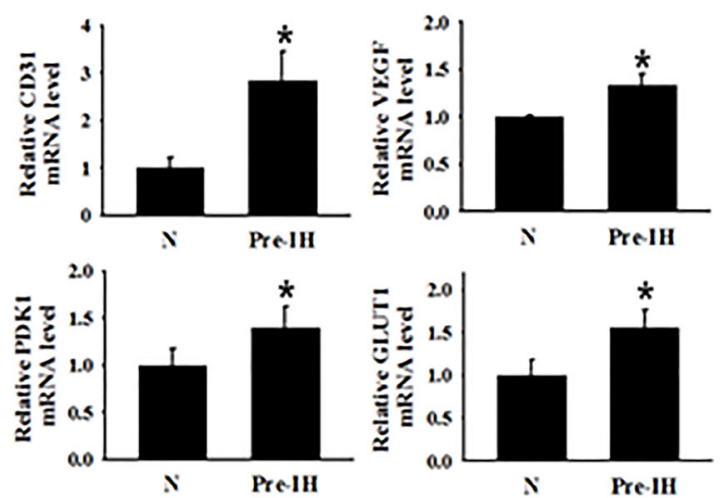

Figure 3: Expression of a cell-proliferation marker, endothelial cell markers, and hypoxia-response transcription factor in tumor tissues from mice in the no-conditioning (N) and pre-intermittent hypoxia conditioning (Pre-IH) groups. (A) Representative immunohistochemical staining images for HIF-1 $\alpha$, PCNA, CD31, and TUNEL-positive nuclei. Tumor tissues were collected on day 19 post-tumor injection. (B) Quantification of the $\%$ of cells staining positive for nuclear HIF- $1 \alpha$, the $\%$ CD 31 -stained area, the \% PCNA-positive cells, and the average number of TUNEL-positive cells per high-power field. (C) Relative CD31, VEGFA, PDK1, and GLUT1 mRNA-expression levels in tumors from mice in the $\mathrm{N}$ and Pre-IH groups. The arrow indicates TUNEL-positive nuclei. Scale bars, $50 \mu \mathrm{m}$ except for CD31 $(100 \mu \mathrm{m}) .{ }^{*} P<0.05$ compared to the $\mathrm{N}$ group, as determined by the Mann-Whitney U test. 
significant difference between the mRNA level of HIF$1 \alpha$ itself in tumors of the IH-2 group, compared with the other two groups (Supplementary Figure 1C), implying that HIF-1 $\alpha$ up-regulation in the IH-2 group was possibly due to increased protein stability, as opposed to elevated protein synthesis.

\section{DISCUSSION}

In this study, we found that $\mathrm{IH}$ increased in vivo tumor growth, both with tumor cells that were preconditioned with $\mathrm{IH}$ and exposed to $\mathrm{IH}$ following tumor injection. Accelerated tumor growth was also associated with HIF-1-dependent hypoxia adaptation.
Hypoxia occurs commonly in solid tumors as the tumors proliferate more than the existing vascular can supply. Thus, adaptive responses to hypoxia (i.e., elevation of oxygen supply or compensation for the loss of energy) are an essential process for tumor growth and survival. HIF-1 is the best-known mediator of adaptive cellular responses to hypoxia and consists of a heterodimer of HIF-1 $\alpha$ and HIF-1 $\beta$, which are basic helix-loop-helix PAS proteins [21]. HIF-1 is hydroxylated by prolyl hydroxylases (PHDs) and degraded through the ubiquitinmediated proteasome pathway under normoxia. Under hypoxia, PHDs are inactivated and HIF-1 $\alpha$ is stabilized in the cytoplasm. Stabilized HIF- $1 \alpha$ undergoes nuclear translocation and dimerizes with HIF-1 $\beta$ [22]. The HIF-
A
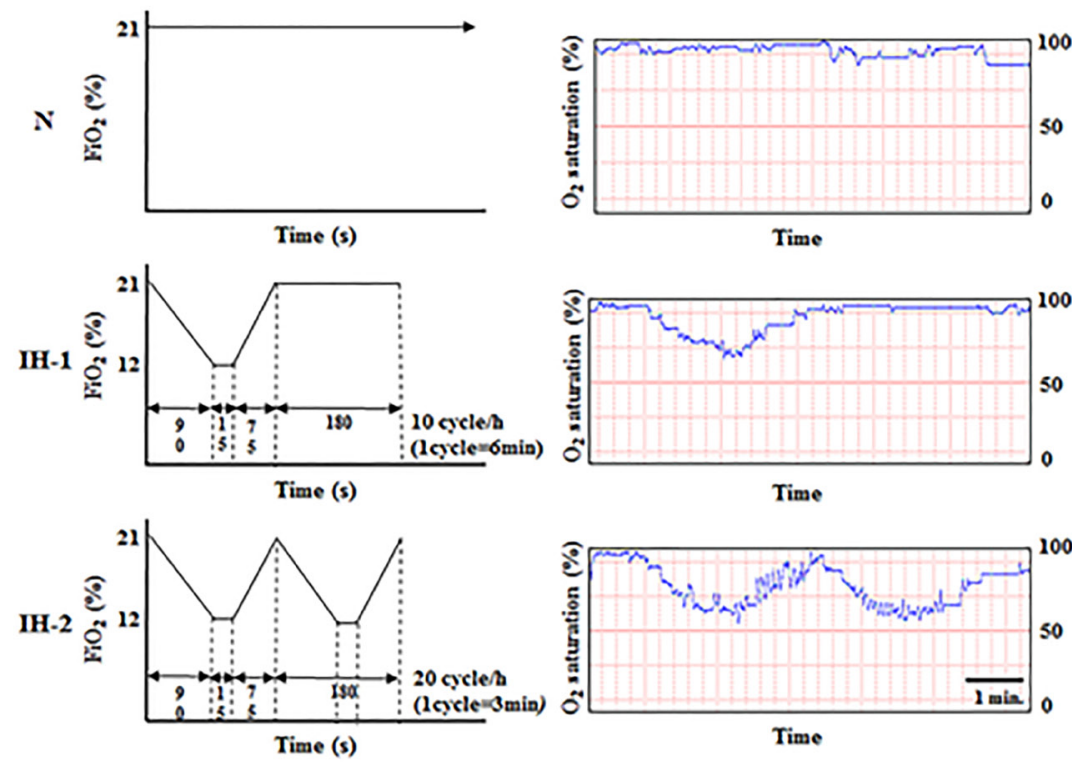

C

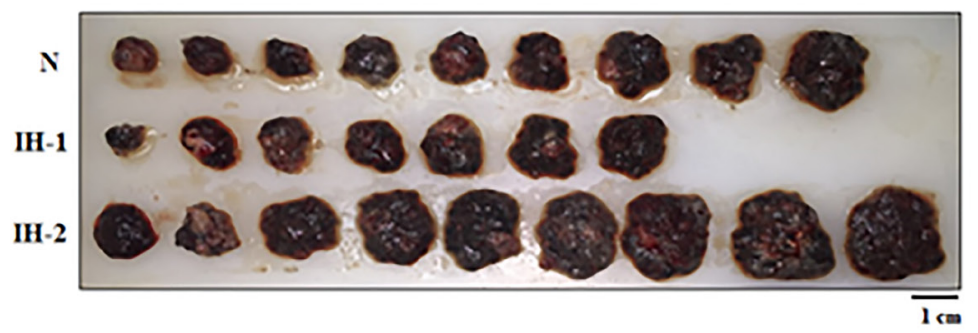

B
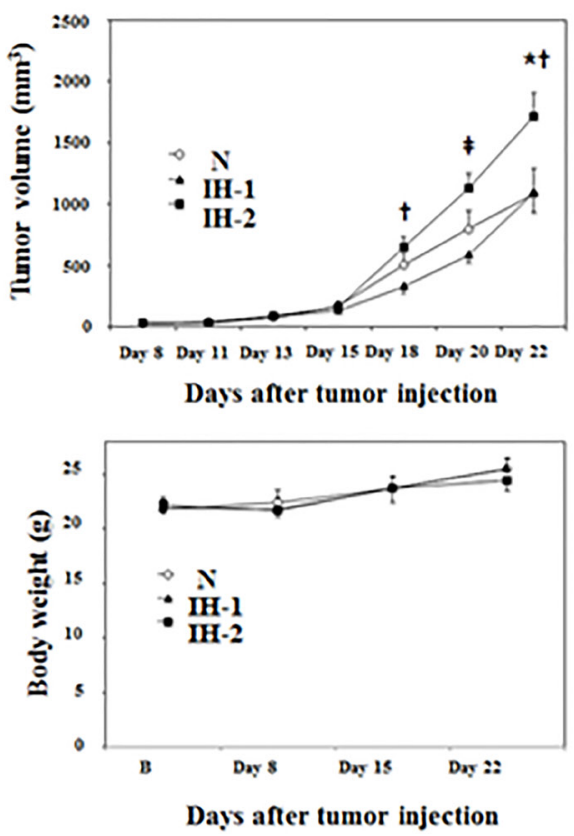

D

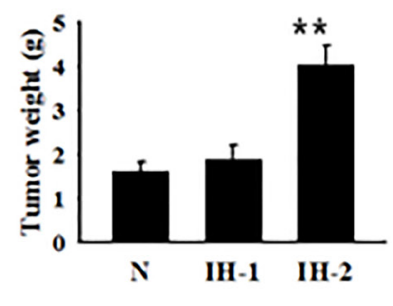

Figure 4: Tumor growth in mice subjected to normoxia (N), intermittent hypoxia-1 (IH-1), or intermittent hypoxia-2 (IH-2) (n = 10/group initially). (A) Time course of oxygen-saturation profiles measured by mouse oximetry in mice during exposure to $\mathrm{N}$, IH-1, or IH-2 conditions. Mice in the $\mathrm{N}$ group were maintained under a constant $\mathrm{FiO}_{2}$ of $21 \%$. The experimental parameters used for 1 cycle of $\mathrm{IH}-1$ were $21 \% \rightarrow 12 \% \mathrm{FiO}_{2}$ for $90 \mathrm{~s}, 12 \% \mathrm{FiO}_{2}$ for $15 \mathrm{~s}, 12 \% \rightarrow 21 \% \mathrm{FiO}_{2}$ for $75 \mathrm{~s}$, and $21 \% \mathrm{FiO}$ for $180 \mathrm{~s}(10 \mathrm{cycles} / \mathrm{h})$. The parameters for 1 cycle of $\mathrm{IH}-2$ were $21 \% \rightarrow 12 \% \mathrm{FiO}_{2}$ for $90 \mathrm{~s}, 12 \% \mathrm{FiO}_{2}$ for $15 \mathrm{~s}$, and $12 \% \rightarrow 21 \% \mathrm{FiO}_{2}$ for $75 \mathrm{~s}(20 \mathrm{cycles} / \mathrm{h}$ ). (B) Changes in tumor volumes and body weights. (C) Comparison of tumor sizes from mice subjected to N, IH-1, or IH-2 conditions. Tumors were excised from mice on day 22 post-tumor injection ( $\mathrm{n}=9$ for $\mathrm{N} ; \mathrm{n}=7$ for $\mathrm{IH}-1$, and $\mathrm{n}=9$ for IH-2). (D) Comparison of tumor weights in the N, IH-1, and IH-2 groups. Data are presented as the mean \pm S.E.M. ${ }^{*} P<0.05$ compared to $\mathrm{N} ;{ }^{\dagger} P<0.05$ compared to IH-1; ${ }^{\ddagger} P<0.01$ compared to IH-1; ${ }^{* *} P<0.01$ compared to $\mathrm{N}$ and IH-1, as determined by the Kruskal-Wallis test, followed by the Mann-Whitney U test. 
$1 \alpha / \beta$ dimer subsequently binds to hypoxia-response elements and modulates the transcription of several target genes, which are favor cancer progression (e.g., angiogenesis, glucose metabolism, cell proliferation, apoptosis, invasion, and metastasis). We found that HIF- $1 \alpha$ and its downstream targets were up-regulated in tumors from mice subjected to 2 different $\mathrm{IH}$ protocols. These findings implied that a series of HIF-1-dependent, hypoxia-adaptation processes may play important roles in cancer progression promoted by IH. In a previous study that investigated the effect of $\mathrm{IH}$ on tumor growth in xenografted mice, circulating VEGF levels in the blood positively correlated with tumor weights [16]. However, no significant difference was observed between tumor VEGF and CD31 expression in mice subjected to IH. HIF$1 \alpha$ expression levels in tumors were also not examined.
Another study provided evidence of increased VEGF expression and angiogenesis in human melanoma-derived tumors in mice, as well as elevated in vitro VEGF secretion in the same tumor cell lines when exposed to cyclic hypoxia [23]. Although the causal role of VEGF, which is elevated by $\mathrm{IH}$, on tumor growth was not elucidated in those studies, it is clear that the upregulation of HIF-1dependent VEGF expression promotes angiogenesis and contributes to tumor growth [24, 25].

While previous findings have shown that $\mathrm{IH}$ promotes cancer cell proliferation, as demonstrated by cell growth assays and cell cycle analysis [26], we did not observe any significant difference in biological behavior such as cell cycle progression or proliferation of melanoma cells that were maintained under normoxia or pre-IH-conditioned. These discrepancies might be due to
A

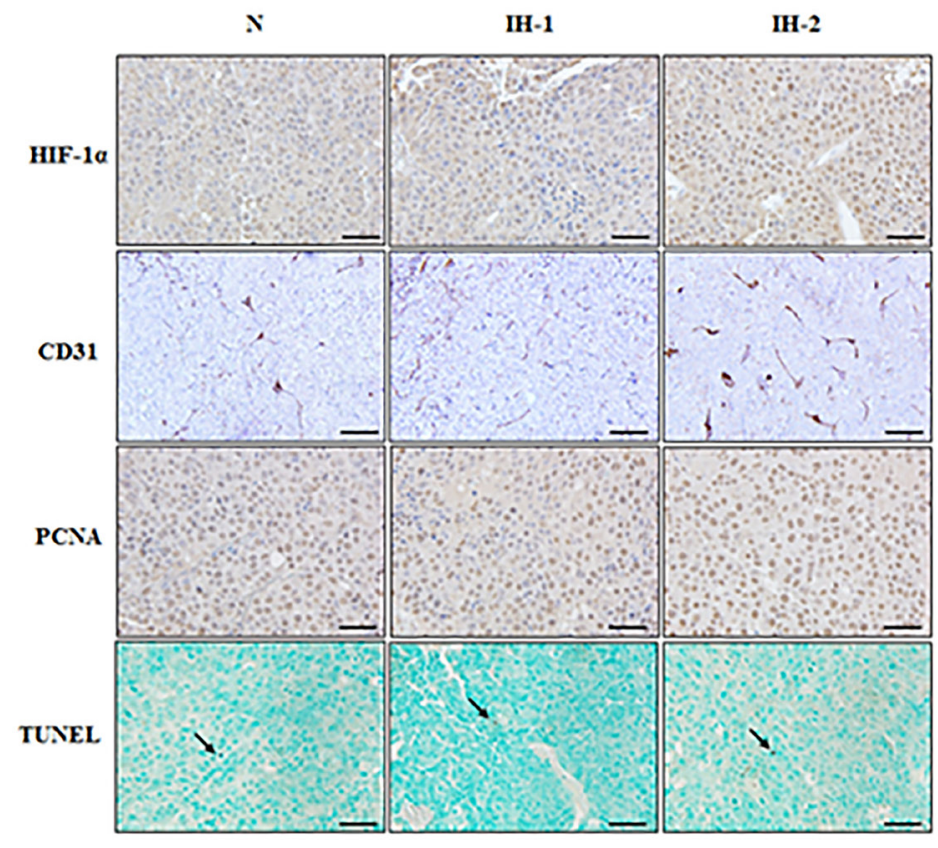

B
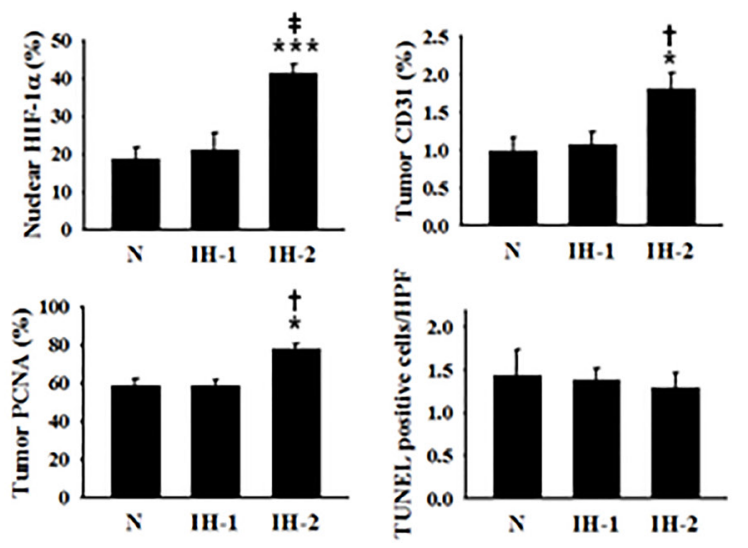

C
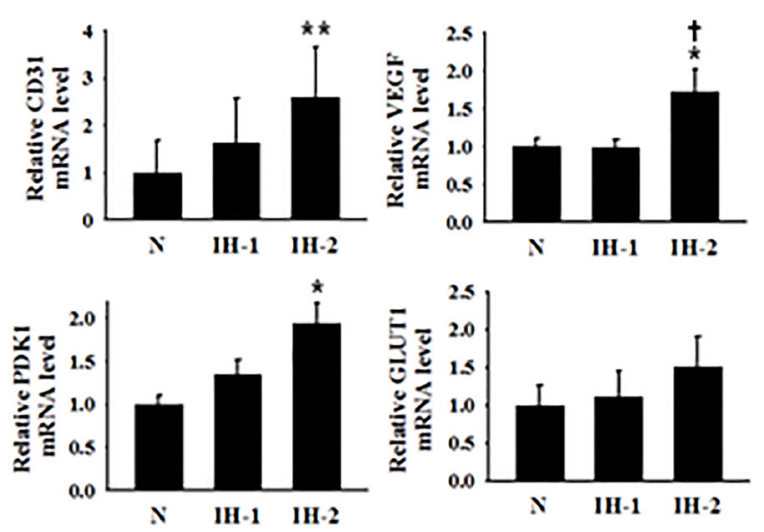

Figure 5: Expression of a cell-proliferation marker, endothelial cell markers, and hypoxia-response transcription factor in tumor tissues from mice subjected to normoxia (N), intermittent hypoxia-1 (IH-1), or intermittent hypoxia-2 (IH-2) conditions. (A) Representative immunohistochemical staining images for HIF-1 $\alpha$, PCNA, CD31, and TUNEL-positive nuclei. Tumor tissues were collected on day 22 post-tumor inoculation. (B) Quantification of the $\%$ cells staining positive for nuclear HIF-1 $\alpha$, the $\%$ CD31-stained area, the \% PCNA-positive cells, and the average number of TUNEL-positive cells/high-power field in the N, IH-1, and IH-2 groups. (C) Relative CD31, VEGFA, PDK1, and GLUT1 mRNA-expression levels in tumors from mice in the N, IH-1, and IH-2 groups. Scale bars, $50 \mu \mathrm{m}$ except for CD31 $(100 \mu \mathrm{m})$. The arrow indicates TUNEL-positive nuclei. ${ }^{*} P<0.05$ compared to the N group; ${ }^{* *} P<0.01$ compared to the $\mathrm{N}$ group; ${ }^{* * *} P<0.001$ compared to the $\mathrm{N}$ group; ${ }^{\dagger} P<0.05$ compared to the IH-1 group; ${ }^{\ddagger} P<0.01$ compared to the IH-1 group, as determined by the Kruskal-Wallis test, followed by the Mann-Whitney U test. 
differences in IH protocols (e.g., frequency and duration), the level of $\mathrm{FiO}_{2}$ used for hypoxia, cell line differences, and co-culture with other immune cells.

We also observed different findings between in vitro and in vivo experiments regarding HIF-1 $\alpha$-dependent hypoxic adaptation and cell-proliferative potential. The underlying mechanism by which hypoxia-adaptive responses and tumor growth in the Pre-IH group were more accelerated in vivo than were those in the $\mathrm{N}$ group is unknown. We speculate that long-term exposure to $\mathrm{IH}$ can make cancer cells acquire a persistent transformation, which is advantageous for adapting or proliferating under in vivo hypoxic environment, possibly through feedforward regulation of biochemical/metabolic pathways or epigenetic modification [27]. This speculation is based partially on the findings that cancer cells pre-exposed to IH showed up-regulated expression of PDK1, revealing that those pre-trained cells were relatively prepared to adapt to IH conditions. This finding implies that when the xenografted cells formed a mass with intermittent hypoxia inside, those cells may overcome IH more easily. Previous results showed that an 8 -week intermittent induction of HIF-1 $\alpha$, whose expression was regulated by tetracycline, promoted the growth or invasion of human glioma cells in mouse brains [27]. In addition, similar to our findings, the long-term intermittent induction of HIF-1 $\alpha$ resulted in lost HIF-1 $\alpha$ expression, but did not influence cell proliferation in vitro. These results cannot be directly compared to our results because HIF-1 $\alpha$ expression was artificially driven (i.e., not by hypoxia), and the cell lines used differed from ours. Nevertheless, the previous data supports our findings that conditions that intermittently increase HIF-1 $\alpha$, such as $\mathrm{IH}$, have long-lasting effects on subsequent malignant tumor progression in vivo.

Interestingly, tumor growth can depend on the frequency of $\mathrm{IH}$; an epidemiological study revealed that only moderate-to-severe OSA (respiratory disturbance index $\geq 15$ ) is independently associated with an increased risk for cancer and mortality [28]. Our data and previous epidemiological findings imply the existence of a threshold of $\mathrm{IH}$ frequency, beyond which malignance occurs more often. However, conflicting results regarding the relationship between the severity of OSA and cancer risk also exist. In a large cohort study conducted to analyze data from 10,149 patients who underwent a sleep study, the severity of OSA was not significantly associated with either the prevalence or incidence of cancer after controlling for possible confounding factors [29].

Factors that can determine the threshold of the malignant phenotype of cancer cells based on $\mathrm{IH}$ are unknown, but reactive oxygen species (ROS) are potential candidate molecules linking these processes. ROS upregulates HIF-1 $\alpha$ expression by stimulating synthesis or increasing its stability through $\mathrm{Ca}^{2+}$-dependent signaling pathways, including those involving phospholipase $\mathrm{C} \gamma$ or protein kinase $\mathrm{C}$ and $\mathrm{mTOR}[30,31]$. In humans, OSA severity positively correlates with oxidative stress parameters $[32,33]$. Therefore, ROS elevation above a threshold level may increase the stability of HIF-1 $\alpha$, thereby augmenting HIF-1-dependent adaptation responses to hypoxia, and contribute to tumor progression under $\mathrm{IH}-2$ conditions. This possibility can be examined in further studies using ROS scavengers or conditional HIF- $1 \alpha$-knockout models.

The alteration of sympathetic outflow might also be a contributing factor for the different malignant phenotypes elicited by these IH protocols. Indeed, data from many epidemiologic and experimental studies have revealed that the $\beta$-adrenergic receptor signaling pathway was involved in the proliferation, metastasis, and angiogenesis of several types of tumors [34-36]. Accumulating evidence has indicated that IH is a powerful sympathetic activator [37, 38]. IH elicits both catecholamine synthesis and release via ROS-dependent tyrosine hydroxylase activation [39, 40]. It appears that HIF- $1 \alpha$ mediates catecholamine-induced tumor malignancy in some types of cancer. Treatment with norepinephrine (NE), a ligand of the adrenergic receptor, up-regulated VEGF expression and angiogenesis in an HIF-1 $\alpha$-dependent manner in human breast, prostate, and hepatocellular cancer cells. However, pretreatment with either $\alpha$ - or $\beta$-adrenergic receptor blockers abolished such induction by NE [41]. Although we did not investigate sympathetic activity at the local or systemic level with the IH protocols designed for the present study, the possibility that catecholamine-associated adrenergic receptor signaling determines malignant phenotypes should be investigated in further studies.

In addition, the increased genomic instability and activation of cancer stem cells (CSCs) might be regarded as possible factors linking $\mathrm{IH}$ with enhanced cancer progression [42]. Those are likely to be caused by impaired activities of enzymes responsible for DNA metabolism [43] and HIF-mediated expression of CSCs factor such as Oct4, c-Myc, Wnt, and Notch [44].

In summary, we observed that both pre-IH conditioning and $\mathrm{IH}$ exposure in tumor-bearing mice promoted tumor growth in vivo, and the accelerated progression was associated with HIF-1-dependent adaptive responses to hypoxia. HIF-1-dependent angiogenesis promotes tumor growth, survival, or metastasis, antiangiogenic therapy, and targeting up-regulated HIF1 expression by $\mathrm{IH}$ could be an attractive therapeutic modality [45]. Further mechanistic studies involving pharmacologic inhibition or genetic manipulation are required to elucidate the exact mechanism by which $\mathrm{IH}$ promotes tumor progression.

\section{MATERIALS AND METHODS}

\section{Cell culture and in vitro preconditioning by IH}

Mouse melanoma (B16F10) cells were obtained from Korean Cell Line Bank (Seoul, Korea) and were 
cultured in 100-mm dishes in Dulbecco's modified Eagle's medium containing 10\% fetal bovine serum, 100 $\mathrm{U} / \mathrm{ml}$ penicillin, and $100 \mu \mathrm{g} / \mathrm{ml}$ streptomycin. Prior to preconditioning, the cells were maintained in a humidified incubator at $37^{\circ} \mathrm{C}$ and $5 \% \mathrm{CO}_{2}$. For Pre-IH experiments, cells were exposed to 12 consecutive cycles of hypoxia for $50 \min \left(1 \% \mathrm{O}_{2}, 5 \% \mathrm{CO}_{2}, 37^{\circ} \mathrm{C}\right.$; balanced $\mathrm{N}_{2}$ and water vapor) followed by $10 \mathrm{~min}$ of normoxia ( $\mathrm{N}$ conditions; $21 \% \mathrm{O}_{2}, 5 \% \mathrm{CO}_{2}, 37^{\circ} \mathrm{C}$; balanced $\mathrm{N}_{2}$ and water vapor) during a day. After completing the 12 cycles, the cells returned to the $\mathrm{N}$ conditions and maintained for $12 \mathrm{~h}$. This procedure was repeated for 7 consecutive days. During this period, the cells were sub-cultured twice after being returned to the $\mathrm{N}$ conditions. $\mathrm{N}$-conditioned cells were continuously maintained in the normoxic chamber during the same period. After 7 days of Pre-IH or $\mathrm{N}$ conditioning, $2 \times 10^{5}$ B16F10 cells in $100 \mu$ of phosphate-buffered saline (PBS) were subcutaneously injected into the backs of mice treated with no hypoxic stimulus. Tumor growth was assessed by externally measuring tumor volumes beginning on day 10 at 2-3 day intervals after the injection of cancer cells. The tumor volume (V) was estimated by externally measuring its length (L) and width $(\mathrm{W})$ with a caliper $\left(\mathrm{V}=\mathrm{W}^{2} \times \mathrm{L} / 2\right)$. On day 19 , the mice were sacrificed and the tumors were excised. The excised tumors were rinsed in cold PBS and processed for histological examinations and gene-expression studies.

\section{In vivo IH model}

Thirty male, 8-week-old C57BL/6 mice (Orient Bio, Seongnam, Korea) were used in this study. All animals were maintained in a temperature-controlled room with alternating 12-h cycles of light and dark (light on at 8:00 a.m.). All experimental procedures were approved by The Institutional Animal Care and Use Committee of the Seoul National University College of Medicine (SNU-1502131). After subcutaneous injection of the B16F10 cells, the animals were randomly divided into 3 groups: 1) $\mathrm{N}$ (n $=10), 2) \mathrm{IH}-1(\mathrm{n}=10)$, and 3$) \mathrm{IH}-2(\mathrm{n}=10)$. IH was applied to the mice on the day after tumor cell injection using an OxyCycler A84XOV gas controller (BioSpherix, NY, USA). The time course of oxygen-saturation profiles in mice was monitored using a MouseOx Plus oximeter (Starr Life Sciences, PA, USA). Mice in the IH-1 group were subjected to $\mathrm{IH}\left(90 \mathrm{~s}\right.$ of $12 \% \mathrm{FiO}_{2}$ followed by $270 \mathrm{~s}$ of $\left.21 \% \mathrm{FiO}_{2},[10 \mathrm{cycles} / \mathrm{h}]\right)$ for $8 \mathrm{~h} /$ day during the light period (09:00-17:00) for 21 consecutive days. Mice in the IH-2 group were subjected to $\mathrm{IH}$ with alternating cycles of $90 \mathrm{~s}\left(12 \% \mathrm{FiO}_{2}\right.$ followed by $90 \mathrm{~s}$ of $21 \% \mathrm{FiO}_{2}$, 20 cycles $/ \mathrm{h}$ ) for the same duration as mice in the IH-1 group. Mice in the $\mathrm{N}$ group were maintained in a chamber supplied with continuously circulating room air. Mice were subcutaneously injected with $1 \times 10^{6}$ B16F10 cells suspended in $100 \mu \mathrm{l}$ of PBS on the back. Tumor growth was assessed beginning on day 8 at 2-3 day intervals after tumor cell injection, using the same method in the preconditioning experiments. On day 22, the mice were sacrificed and the tumors were excised and weighed. The excised tumors were processed as performed in the preconditioning experiment.

\section{Cell cycle analysis and BrdU-based cell proliferation assay}

Cell cycle analysis was performed in normoxic or preconditioned B16F10 cells for 7 days. Before treatment for cell cycle analysis, preconditioned or non-conditioned $\mathrm{B} 16 \mathrm{~F} 10$ cells were incubated under normoxia at $37^{\circ} \mathrm{C}$ overnight. Cells were then harvested, washed with PBS, and fixed in cold $75 \%$ ethanol overnight at $-20^{\circ} \mathrm{C}$. After washing and re-suspension in PBS, the fixed cells were treated with RNase A (Sigma-Aldrich) for $40 \mathrm{~min}$ at $37^{\circ} \mathrm{C}$. Propidium iodide was then added to each sample. Cell proliferation was determined using the FITC BrdU Kit (Becton Dickinson [BD], USA), according to the manufacturer's instructions, at the same time point used for cell cycle analysis. Briefly, $1 \times 10^{6} \mathrm{~B} 16 \mathrm{~F} 10$ cells grown in $60-\mathrm{mm}$ dishes were treated with BrdU-incorporation solution and incubated for $1 \mathrm{~h}$ at $37^{\circ} \mathrm{C}$, followed by fixation and permeabilization. After DNase treatment to expose the incorporated $\mathrm{BrdU}$ for $1 \mathrm{~h}$ at $37^{\circ} \mathrm{C}$, the fixed cells were incubated with an anti-BrdU antibody for $20 \mathrm{~min}$ at room temperature. Total DNA levels were determined by 7-AAD staining. BrdU-incorporated or 7-AAD stained cells were analyzed on a BD FACSCanto ${ }^{\mathrm{TM}}$ flow cytometer (BD Bioscience, USA), and DNA histograms were constructed using BD FACSDiva software, version 8.0.1.

\section{Immunohistochemical staining and TUNEL assay}

For immunohistochemical staining, tumors excised from mice were fixed in $4 \%$ paraformaldehyde at 4 for 24 and embedded in paraffin. For antigen retrieval, $4-\mu \mathrm{m}$ thick sections were autoclaved at $121^{\circ} \mathrm{C}$ for $10 \mathrm{~min}$ in $100 \mathrm{mmol} / \mathrm{L}$ citrate buffer (pH 6.0; Dako, Glostrup, Denmark). After treatment with 3\% hydrogen peroxide in methanol for $10 \mathrm{~min}$ in a moisture chamber, the sections were incubated with $2 \%$ blocking serum for $1 \mathrm{~h}$ at room temperature and then incubated overnight with a primary anti-HIF-1 $\alpha$ (1: 100; Novus Biologicals, CO, USA), anti-CD31 (1: 200; Thermo Scientific, USA), or antiPCNA (1: 1000; Santa Cruz Biotechnology, Inc., USA) antibody at $4^{\circ} \mathrm{C}$. After overnight incubation with the primary antibodies, the sections were incubated for $1 \mathrm{~h}$ with a secondary antibody provided in GBI Polink HRP Kit (Golden Bridge International, Inc., USA). Then, the sections were developed using 3,3'-diaminobenzidine. Hematoxylin was employed for counter staining (Vector Laboratories, Inc., CA, USA). Sections were analyzed with a bright-field microscope (BX-51; Olympus, Tokyo, 
Japan) equipped with a DP70 camera (Olympus). To evaluate the results of IHC staining, 5 random high-power fields per slide (HPF; X 400) with no necrotic areas were acquired from all mice (10 tumors in the $\mathrm{N}$ group and 10 tumors in the Pre-IH group; or 9 tumors in the $\mathrm{N}$ group, 7 tumors in the $\mathrm{IH}-1$ group, and 9 tumors in the $\mathrm{IH}-2$ group), and the percentages of cells showing positive nuclear HIF$1 \alpha$ or PCNA expression, and the \%CD31-positive areas were determined using the Image J program. IHC images were analyzed under blind conditions. These levels are presented as the mean values of the images. To determine the degree of apoptosis, we performed TUNEL assay using the APO-BRDU-IHC Kit (Novus Biologicals, CO, USA), according to the manufacturer's protocols. The extent of apoptosis was expressed as the mean number of TUNELpositive nuclei per HPF.

\section{Western blot analysis}

Cells were rinsed in ice-cold PBS and harvested in lysis buffer. Equivalent amounts of protein were separated by $8 \%$ SDS-PAGE and transferred to a polyvinylidene membrane (Millipore, MA, USA). Membranes were blocked with $5 \%$ nonfat dried milk for $1 \mathrm{~h}$ at room temperature; incubated at $4^{\circ} \mathrm{C}$ overnight with either a rabbit polyclonal anti-HIF-1 $\alpha$ antibody (1: 1000; customized), a rabbit polyclonal anti-HIF- $2 \alpha$ antibody (1: 1000; Cell Signaling Technology, MA, USA), or a rabbit polyclonal anti-PDK1 antibody (1: 1000; Enzo Life Sciences Inc., NY, USA); and then incubated for $1 \mathrm{~h}$ with a horseradish peroxidase-conjugated secondary antibody (1: 5000). Signals were detected using the Luminata ${ }^{\mathrm{TM}}$ Crescendo Western HRP Substrate solution (Millipore, MA, USA). $\beta$-tubulin (1: 5000; Santa Cruz Biotechnology, Inc.) was detected as a loading control.

\section{RNA isolation and qRT-PCR}

Total RNA from excised tumor tissue was extracted using the TRIzol reagent (Ambion). One microgram of total RNA was reverse transcribed into complementary DNA using the Tetro cDNA Synthesis Kit (Bioline, London, UK), according to the manufacturer's instructions. qRT-PCR was performed using an iQ5 Real-Time PCR System (Bio-Rad Laboratories, Hercules, CA, USA). All reactions were performed in duplicate, and the specificity was examined by melting-curve analysis. Target-specific primers with the following sequences were used for amplification: HIF 1A, F: 5'-CTGCAGGGTGAAGAATTACT-3' and R: 5'-GTTTGTGCAGTATTGTAGCC-3'; VEGFA, F: 5'-TGC AGGCTGCTGTAACGATG-3' and R: 5'-CCTCGG CTTGTCACATTTTTCT-3'; PECAM1 (CD31), F: 5'-TC ACCATCAACAGCATCCAT-3' and R: 5'-GGTGCTGA GACCTGCTTTTC-3'; PDK1, F: 5'-CAACTACCCTTGG TATGGTATGGG-3' and R: 5'-CGTGGGAGATAAG
AAGACCATCTG-3'; GLUT1, F: 5'-AACATGGAACC ACCGCTACG-3' and R: 5'-GTGGTGAGTGTGGTG GATGG-3'; ACTB, F: 5'-GGCTGTATTCCCCTCC ATCG-3' and R: 5'-CCAGTTGGTAACAATGCCATGT-3'. The 2-delta-delta comparative cycle threshold $(\mathrm{Ct})$ method was used to compare the relative gene-expression levels, which were normalized against $\beta$-actin mRNA expression.

\section{Statistical analysis}

All data were expressed as the mean \pm standard error of the mean (S.E.M). Differences in the means were evaluated using the non-parametric Kruskal-Wallis test. Mann-Whitney $U$-tests were used for the post-hoc analyses and comparisons of variables between 2 independent groups. All statistical analyses were performed using IBM SPSS, version 20.0 (SPSS; Chicago, IL, USA), and $P<$ 0.05 was considered to indicate statistical significance.

\section{Author contributions}

Conception and design: DWY and HWS.

Validation of methodology: DWY, DHS, JYK, and MGL.

Acquisition of data: DWY, DHS, SM, JYK, MGL, and RK.

Analysis and interpretation of data: DWY, DHS, JWP, and HWS.

Writing, review, and revision of the manuscript: DWY and HWS.

Study supervision: JWP, CHC, and HWS.

\section{CONFLICTS OF INTEREST}

The authors have no conflicts of interest to declare.

\section{FUNDING}

This work was supported by a grant from the Korean Health Technology R\&D Project, Ministry of Health and Welfare, Republic of Korea (HI14C2133) and by grant no. 0320160110 from the SNUH Research Fund. D.W. Yoon received a scholarship from the BK21-plus education program provided by the National Research Foundation of Korea.

\section{REFERENCES}

1. Nieto FJ, Young TB, Lind BK, Shahar E, Samet JM, Redline S, D'Agostino RB, Newman AB, Lebowitz MD, Pickering TG. Association of sleep-disordered breathing, sleep apnea, and hypertension in a large community-based study. Sleep Heart Health Study. JAMA. 2000; 283:1829-1836.

2. Shahar E, Whitney CW, Redline S, Lee ET, Newman AB, Nieto FJ, O'Connor GT, Boland LL, Schwartz JE, Samet 
JM. Sleep-disordered breathing and cardiovascular disease: cross-sectional results of the Sleep Heart Health Study. Am J Respir Crit Care Med. 2001; 163:19-25.

3. Adams RJ, Appleton SL, Vakulin A, Hanly PJ, McDonald SP, Martin SA, Lang CJ, Taylor AW, McEvoy RD, Antic NA, Catcheside PG, Vincent AD, Wittert GA. Association of kidney disease with obstructive sleep apnea in a population study of men. Sleep. 2016 Sep 9. [Epub ahead of print].

4. Cadby G, McArdle N, Briffa T, Hillman DR, Simpson L, Knuiman M, Hung J. Severity of OSA is an independent predictor of incident atrial fibrillation hospitalization in a large sleep-clinic cohort. Chest. 2015; 148:945-952.

5. Einvik G, Rosjo H, Randby A, Namtvedt SK, HrubosStrom H, Brynildsen J, Somers VK, Omland T. Severity of obstructive sleep apnea is associated with cardiac troponin I concentrations in a community-based sample: data from the Akershus Sleep Apnea Project. Sleep. 2014; 37:1111-1116, 1116A-1116B.

6. Pujante P, Abreu C, Moreno J, Barrero EA, Azcarate P, Campo A, Urrestarazu E, Silva C, Maria JG, Tebar J, Fruhbeck G, Salvador J. Obstructive sleep apnea severity is associated with left ventricular mass independent of other cardiovascular risk factors in morbid obesity. J Clin Sleep Med. 2013; 9:1165-1171.

7. Nieto FJ, Peppard PE, Young T, Finn L, Hla KM, Farre R. Sleep-disordered breathing and cancer mortality: results from the Wisconsin Sleep Cohort Study. Am J Respir Crit Care Med. 2012; 186:190-194.

8. Campos-Rodriguez F, Martinez-Garcia MA, Martinez M, Duran-Cantolla J, Pena Mde L, Masdeu MJ, Gonzalez M, Campo F, Gallego I, Marin JM, Barbe F, Montserrat JM, Farre R, Spanish Sleep N. Association between obstructive sleep apnea and cancer incidence in a large multicenter Spanish cohort. Am J Respir Crit Care Med. 2013; 187:99-105.

9. Gozal D, Ham SA, Mokhlesi B. Sleep apnea and cancer: analysis of a nationwide population sample. Sleep. 2016; 39:1493-1500.

10. Harris AL. Hypoxia--a key regulatory factor in tumour growth. Nat Rev Cancer. 2002; 2:38-47.

11. Semenza GL. HIF-1: using two hands to flip the angiogenic switch. Cancer Metastasis Rev. 2000; 19:59-65.

12. Ljungkvist AS, Bussink J, Kaanders JH, van der Kogel AJ. Dynamics of tumor hypoxia measured with bioreductive hypoxic cell markers. Radiat Res. 2007; 167:127-145.

13. Chaplin DJ, Hill SA. Temporal heterogeneity in microregional erythrocyte flux in experimental solid tumours. Br J Cancer. 1995; 71:1210-1213.

14. Malec V, Gottschald OR, Li S, Rose F, Seeger W, Hanze J. HIF-1 alpha signaling is augmented during intermittent hypoxia by induction of the Nrf2 pathway in NOX1expressing adenocarcinoma A549 cells. Free Radic Biol Med. 2010; 48:1626-1635.
15. Martinive P, Defresne F, Quaghebeur E, Daneau G, Crokart N, Gregoire V, Gallez B, Dessy C, Feron O. Impact of cyclic hypoxia on HIF-1alpha regulation in endothelial cells--new insights for anti-tumor treatments. FEBS J. 2009; 276:509-518.

16. Almendros I, Montserrat JM, Torres M, Bonsignore MR, Chimenti L, Navajas D, Farre R. Obesity, intermittent hypoxia increase tumor growth in a mouse model of sleep apnea. Sleep Med. 2012; 13:1254-1260.

17. Almendros I, Montserrat JM, Ramirez J, Torres M, DuranCantolla J, Navajas D, Farre R. Intermittent hypoxia enhances cancer progression in a mouse model of sleep apnoea. Eur Respir J. 2012; 39:215-217.

18. Almendros I, Montserrat JM, Torres M, Dalmases M, Cabanas ML, Campos-Rodriguez F, Navajas D, Farre R. Intermittent hypoxia increases melanoma metastasis to the lung in a mouse model of sleep apnea. Respir Physiol Neurobiol. 2013; 186:303-307.

19. Almendros I, Wang Y, Becker L, Lennon FE, Zheng J, Coats BR, Schoenfelt KS, Carreras A, Hakim F, Zhang SX, Farre R, Gozal D. Intermittent hypoxia-induced changes in tumor-associated macrophages and tumor malignancy in a mouse model of sleep apnea. Am J Respir Crit Care Med. 2014; 189:593-601.

20. Herlyn M, Fukunaga-Kalabis M. What is a good model for melanoma? J Invest Dermatol. 2010; 130:911-912.

21. Wang GL, Jiang BH, Rue EA, Semenza GL. Hypoxiainducible factor 1 is a basic-helix-loop-helix-PAS heterodimer regulated by cellular $\mathrm{O} 2$ tension. Proc Natl Acad Sci U S A. 1995; 92:5510-5514.

22. Kaelin WG. Proline hydroxylation and gene expression. Annu Rev Biochem. 2005; 74:115-128.

23. Rofstad EK, Gaustad JV, Egeland TA, Mathiesen B, Galappathi K. Tumors exposed to acute cyclic hypoxic stress show enhanced angiogenesis, perfusion and metastatic dissemination. Int J Cancer. 2010; 127:1535-1546.

24. Eltzschig HK, Carmeliet P. Hypoxia and inflammation. N Engl J Med. 2011; 364:656-665.

25. Pugh CW, Ratcliffe PJ. Regulation of angiogenesis by hypoxia: role of the HIF system. Nat Med. 2003; 9:677-684.

26. Liu Y, Song X, Wang X, Wei L, Liu X, Yuan S, Lv L. Effect of chronic intermittent hypoxia on biological behavior and hypoxia-associated gene expression in lung cancer cells. J Cell Biochem. 2010; 111:554-563.

27. Choi H, Gillespie DL, Berg S, Rice C, Couldwell S, Gu J, Colman H, Jensen RL, Huang LE. Intermittent induction of HIF-1alpha produces lasting effects on malignant progression independent of its continued expression. PLoS One. 2015; 10:e0125125.

28. Marshall NS, Wong KK, Cullen SR, Knuiman MW, Grunstein RR. Sleep apnea and 20-year follow-up for allcause mortality, stroke, and cancer incidence and mortality 
in the Busselton Health Study cohort. J Clin Sleep Med. 2014; 10:355-362.

29. Kendzerska T, Leung RS, Hawker G, Tomlinson G, Gershon AS. Obstructive sleep apnea and the prevalence and incidence of cancer. CMAJ. 2014; 186:985-992.

30. Yuan G, Nanduri J, Bhasker CR, Semenza GL, Prabhakar NR. Ca2+/calmodulin kinase-dependent activation of hypoxia inducible factor 1 transcriptional activity in cells subjected to intermittent hypoxia. J Biol Chem. 2005; 280:4321-4328.

31. Yuan G, Nanduri J, Khan S, Semenza GL, Prabhakar NR. Induction of HIF-1alpha expression by intermittent hypoxia: involvement of NADPH oxidase, Ca2+ signaling, prolyl hydroxylases, and mTOR. J Cell Physiol. 2008; 217:674-685.

32. Franco CM, Lima AM, Ataide L Jr, Lins OG, Castro CM, Bezerra AA, de Oliveira MF, Oliveira JR. Obstructive sleep apnea severity correlates with cellular and plasma oxidative stress parameters and affective symptoms. J Mol Neurosci. 2012; 47:300-310.

33. Villa MP, Supino MC, Fedeli S, Rabasco J, Vitelli O, Del Pozzo M, Gentile G, Lionetto L, Barreto M, Simmaco M. Urinary concentration of 8-isoprostane as marker of severity of pediatric OSAS. Sleep Breath. 2014; 18:723-729.

34. De Giorgi V GM, Gandini S, Benemei S, Lotti T, Marchionni N, Geppetti P. Treatment with $\beta$-blockers and reduced disease progression in patients with thick melanoma. Arch Intern Med. 2011; 171:779-781

35. Lang K, Drell TL $4^{\text {th }}$, Lindecke A, Niggemann B, Kaltschmidt C, Zaenker KS, Entschladen F. Induction of a metastatogenic tumor cell type by neurotransmitters and its pharmacological inhibition by established drugs. Int $\mathbf{J}$ Cancer. 2004; 112:231-238.

36. Powe DG, Voss MJ, Zanker KS, Habashy HO, Green AR, Ellis IO, Entschladen F. Beta-blocker drug therapy reduces secondary cancer formation in breast cancer and improves cancer specific survival. Oncotarget. 2010; 1:628-638. doi: 10.18632/oncotarget.101009.

37. Prabhakar NR, Kumar GK, Peng YJ. Sympatho-adrenal activation by chronic intermittent hypoxia. J Appl Physiol (1985). 2012; 113:1304-1310.

38. Zoccal DB, Bonagamba LG, Oliveira FR, AntunesRodrigues J, Machado BH. Increased sympathetic activity in rats submitted to chronic intermittent hypoxia. Exp Physiol. 2007; 92:79-85.

39. Kumar GK, Rai V, Sharma SD, Ramakrishnan DP, Peng YJ, Souvannakitti D, Prabhakar NR. Chronic intermittent hypoxia induces hypoxia-evoked catecholamine efflux in adult rat adrenal medulla via oxidative stress. J Physiol. 2006; 575:229-239.

40. Raghuraman G, Rai V, Peng YJ, Prabhakar NR, Kumar GK. Pattern-specific sustained activation of tyrosine hydroxylase by intermittent hypoxia: role of reactive oxygen speciesdependent downregulation of protein phosphatase $2 \mathrm{~A}$ and upregulation of protein kinases. Antioxid Redox Signal. 2009; 11:1777-1789.

41. Park SY, Kang JH, Jeong KJ, Lee J, Han JW, Choi WS, Kim YK, Kang J, Park CG, Lee HY. Norepinephrine induces VEGF expression and angiogenesis by a hypoxia-inducible factor-1alpha protein-dependent mechanism. Int J Cancer. 2011; 128:2306-2316.

42. Kukwa W, Migacz E, Druc K, Grzesiuk E, Czarnecka AM. Obstructive sleep apnea and cancer: effects of intermittent hypoxia? Future Oncol. 2015; 11:3285-3298.

43. Toffoli S, Michiels C. Intermittent hypoxia is a key regulator of cancer cell and endothelial cell interplay in tumours. FEBS J. 2008; 275:2991-3002.

44. Sun Q, Li X, Lu X, Di B. Cancer stem cells may be mostly maintained by fluctuating hypoxia. Med Hypotheses. 2011; 76:471-473.

45. Blagosklonny MV. Antiangiogenic therapy and tumor progression. Cancer Cell. 2004; 5:13-17. 